\title{
Simulation interprofessionnelle en visio-conférence
}

\section{Interprofessional telesimulation}

\author{
Patricia PICCHIOTTINO ${ }^{1,2, *}$, Adeline PAIGNON ${ }^{1,2}$, Barbara BACHMANN $^{2}$, et Maud ELMALEH ${ }^{1,2}$ \\ 1 Centre interprofessionnel de simulation (CiS), Haute École de Santé, Genève, Suisse \\ ${ }^{2}$ HES-SO Haute école spécialisée de Suisse Occidentale, Haute école de santé, Genève, Suisse
}

Manuscrit reçu le 19 avril 2020 ; accepté pour publication le 11 juin 2020

\section{Contexte}

La formation interprofessionnelle a été mise en place à Genève pour répondre aux défis de notre système de santé : amélioration de la qualité des soins et de la sécurité des patients, évolution démographique, évolution des rôles des professionnels de la santé. Ces défis imposent une transformation de l'organisation des soins et de la formation. Le Centre interprofessionnel de simulation (CiS) a été créé pour développer les compétences interprofessionnelles tout au long du continuum des formations prégraduées, postgraduées et continues, en utilisant comme principale modalité pédagogique la simulation [1]. Les formations interprofessionnelles au CiS sont élaborées et dispensées selon une approche par compétences. Elles s'appuient sur des outils d'amélioration de la performance du travail en équipe et de la sécurité des patients, tels que le modèle TeamSTEPPS ${ }^{\circledR}[2]$.

En formation prégraduée, trois modules interprofessionnels impliquent environ 1500 étudiants de sept filières de formation: médecins, pharmacien-nes, infirmier-es, sages-femmes, technicien-nes en radiologie médicale, diététicien-nes, physiothérapeutes [3].

Parmi ces trois modules, le second s'appuie essentiellement sur des simulations interprofessionnelles. Il s'adresse à des étudiants en milieu de formation $\left(2^{\mathrm{e}}\right.$ année de bachelier pour les soignants et $1-2^{\mathrm{e}}$ de master pour les médecins). Les buts du module sont notamment, à travers des situations cliniques proches de la réalité professionnelle, de parvenir à une représentation partagée de situations cliniques, de négocier en équipe des objectifs de prise en charge / intervention et d'exercer des techniques de communication.

\footnotetext{
*Correspondance et offprints : Patricia PICCHIOTTINO. Centre Interprofessionnel de Simulation, CMU 1, rue Michel Servet, 1211 Genève 4, Suisse.

Mailto: patricia.picchiottino@hesge.ch.
}

\section{Problématique}

Les mesures de confinement liées à l'épidémie du Covid-19 ont imposé l'arrêt subit de l'ensemble des activités d'enseignement en présence. Cela a remis en question les simulations s'adressant aux étudiants en médecine, sages-femmes et technicien-nes en radiologie médicale (TRM) qui devaient être mis en situation sur la thématique du syndrome de détresse respiratoire du nouveau-né. Le scénario impliquait une radio du thorax pour confirmer la suspicion d'un wet lung ou d'un pneumothorax. Cette simulation concernait 80 étudiants, répartis en six groupes de 12-13 étudiants sur trois demijournées. Chaque groupe devait participer à une simulation de deux heures comprenant un briefing, deux courtes simulations d'environ 10 minutes et suivies chacune d'un débriefing animé par deux tuteurs de profession différente.

La première semaine de fermeture des institutions a empêché la tenue de la première demi-journée de simulation, au profit d'un réaménagement des dispositifs de formation. Par conséquent, nous ne disposions plus que de deux demi-journées pour mettre en place un nouveau design pour ces 80 étudiants.

Le défi était donc d'atteindre les objectifs pédagogiques initiaux dans un temps réduit et à distance.

\section{Ce que nous avons fait}

Nous avons souhaité maintenir la modalité de simulation et l'adapter à un dispositif synchrone à distance [4].

Dans un premier temps, nous avons redimensionné les objectifs pour les rendre plus opérationnels et atteignables dans un temps réduit. Initialement, les objectifs étaient les suivants: délimiter les compétences, rôles et responsabilités des différents acteurs de la prise en charge, exercer la communication entre professionnels pour la réalisation de l'objectif thérapeutique et prendre en compte le contexte dans la prise en charge. Nous avons choisi de conserver uniquement les deux premiers objectifs, en précisant le 
second ainsi: mettre en œuvre une communication structurée pour transmettre les informations critiques en utilisant l'outil SCAR (Situation-Contexte-AppréciationRecommandation).

Le scénario initial a ensuite été modifié pour transposer les enjeux de façon réaliste à distance. Chaque professionnel était situé dans son environnement de travail spécifique et devait communiquer avec les autres par téléphone, chacun avec des préoccupations différentes : le TRM attendu pour un autre examen, la sage-femme inquiète de la clinique du nouveau-né, le médecin débutant son service. Les consignes ont été adaptées afin d'atteindre les objectifs visés. La sage-femme devait transmettre par téléphone les éléments pertinents au médecin et décider avec lui de la prise en charge du nouveau-né. Ils devaient ensuite organiser la radio avec le TRM pour confirmer le diagnostic.

Le débriefing interprofessionnel devait être ciblé sur trois éléments principaux: le partage des hypothèses diagnostiques et du degré d'urgence de la situation entre les trois professionnels, l'utilisation de l'outil SCAR pour les transmissions et plus largement sur les besoins exprimés par chaque professionnel pour prendre en charge le nouveau-né.

Les étudiants ont ensuite été répartis en 16 groupes de cinq étudiants, accompagnés par deux tuteurs. Chaque groupe a disposé de 30 minutes. Les étudiants ont reçu par courriel un scénario spécifique à leur profession ainsi qu'un lien pour se connecter au logiciel Zoom ${ }^{\circledR}$ permettant de se retrouver en visio-conférence de façon synchrone. Après la phase d'accueil et de briefing par les tuteurs, une vidéo d'une minute d'un nouveau-né en détresse respiratoire leur a été présentée afin de faciliter l'immersion. La séquence s'est ensuite déroulée comme la simulation en présence et s'est terminée par un débriefing.

\section{Ce que nous avons observé}

Au terme des deux demi-journées de formation, il a été constaté que ce format de simulation à distance a répondu de manière adéquate aux objectifs fixés. En effet, le débriefing, phase reconnue d'apprentissage et de réflexivité, a permis de travailler la performance d'équipe en reprenant le partage de la représentation sur la situation clinique, les objectifs et les besoins des professionnels pour la prise en charge, ainsi que la mise en œuvre d'une méthode de communication structurée dans les transmissions (SCAR).

En ce qui concerne le partage des hypothèses diagnostiques, il s'est avéré que le TRM était peu impliqué et uniquement informé de la prescription de l'examen radiologique. Le rôle du TRM dans l'alignement entre l'hypothèse diagnostique et l'examen, et donc de l'importance d'être informé sur ce qui est recherché, a été régulièrement soulevé comme un élément important de sécurité et d'efficience dans la prise en charge.
En ce qui concerne l'identification des besoins des différents professionnels, il a été soulevé l'importance de les exprimer explicitement en équipe pour mieux anticiper les éventuelles difficultés. Dans notre situation, cela concernait notamment le besoin d'assistance pendant la radio, la connaissance du contexte de l'examen, la possibilité d'appliquer les règles de radioprotection.

L'outil SCAR a pu être systématiquement revu et entraîné. Son intérêt dans une transmission urgente a principalement été mis en évidence dans ses dimensions «Appréciation» et «Recommandation». Ainsi, l'appréciation du degré d'urgence de la situation et sa traduction dans une recommandation concrète ont très rarement été réalisées par les étudiants. La perception de l'urgence, peu argumentée, a rarement débouché sur une demande d'intervention dans une fenêtre de temps précise. Recherchée dans le débriefing, la perception du degré d'urgence de l'intervention pouvait aller de 15 minutes à une demijournée selon les étudiants. Ce point s'est révélé central, une notion d'urgence non explicitée représentant un réel danger en situation réelle.

D'autres points ont encore été discutés, comme le tri des informations utiles à communiquer en fonction de l'interlocuteur et du degré d'urgence, ou le risque lié à l'utilisation des jargons professionnels.

\section{Points forts et points faibles}

Mise à part l'énergie dont l'équipe a fait preuve pour construire une solution alternative en une semaine, le principal point fort a été d'avoir su recentrer l'exercice sur l'essentiel. En effet, les tuteurs ont été surpris de la richesse du débriefing dans un temps si court. Il semble que sur ce point, le dispositif ait gagné en efficience. Plus ciblé, les points prévus ont été systématiquement abordés. Le «take home message » des étudiants en fin de session a dans tous les cas relevé l'adéquation entre les objectifs et les points retenus.

Un autre point fort est la simplicité de ce format. Par le biais d'un courriel contenant les consignes et un lien d'accès au logiciel de visio-conférence (ici, Zoom ${ }^{\circledR}$ ), tous les étudiants ont accédé facilement à la simulation depuis chez eux via un ordinateur ou leur smartphone. Cela présuppose néanmoins de vérifier que chaque étudiant dispose d'une connexion internet et du matériel adéquat.

Un autre avantage réside dans la possibilité de mobiliser les étudiants, sans les inconvénients logistiques des déplacements, des disponibilités de salles et de matériel. À relever que les étudiants se sont montrés très impliqués et participatifs, montrant beaucoup d'enthousiasme à tester ce nouveau format.

Le principal point faible réside dans le degré de réalisme moindre de la mise en situation, qui limite le degré d'immersion. Cela a révélé l'importance du briefing de départ pour permettre, malgré les limites, la réalisation des consignes dans un niveau de réalisme suffisant pour effectuer la tâche prescrite. 
Le dernier point faible relève du niveau de sécurité relative des données avec le logiciel Zoom ${ }^{\circledR}$.

\section{Conclusion et perspectives}

Dans le contexte de confinement, nous avons eu l'opportunité de mettre en œuvre des simulations interprofessionnelles à distance. Malgré quelques limites, elles ont permis d'atteindre de façon efficiente certains objectifs interprofessionnels. Ces résultats ouvrent la perspective de renouveler l'expérience pour l'entraînement à d'autres outils de communication ou pour l'entraînement à des téléconsultations en équipe interprofessionnelle. Ce type de session courte pourrait s'intégrer dans des dispositifs de formation à distance en e-learning. Il conviendra alors de penser des scénarii réalistes d'une situation de prise en soin à distance afin de maximiser l'immersion des étudiants dans ces simulations.

\section{Références}

1. Motola I, Devine LA, Chung HS, Sullivan JE, Issenberg SB. Simulation in healthcare education: A best evidence practical guide. AMEE Guide No. 82. Med Teach 2013;35:e1511-1530.

2. King HB, Battles J, Baker DP, Alonso A, Salas E, Webster J, et al. TeamSTEPPS ${ }^{\mathrm{TM}}$ : Team Strategies and Tools to Enhance Performance and Patient Safety. In: Henriksen K, Battles JB, Keyes MA, Grady ML (eds). Advances in Patient Safety: New Directions and Alternative Approaches (Vol. 3: Performance and Tools), Rockville (MD): Agency for Healthcare Research and Quality (US), 2008:1-20.

3. Van Gessel E, Picchiottino P, Doureradjam R, Nendaz M, Mèche $\mathrm{P}$. Interprofessional training: Start with the youngest! A program for undergraduate healthcare students in Geneva, Switzerland. Med Teach 2018;40:595-9.

4. McCoy CE, Sayegh J, Alrabah R, Yarris LM. Telesimulation: An innovative tool for health professions education. AEM Education and Training 2017;1:132-6.

Citation de l'article : Picchiottino P., Paignon A., Bachmann B., Elmaleh M. Simulation interprofessionnelle en visio-conférence. Pédagogie Médicale 2020:21;211-213 